\begin{tabular}{|c|c|c|c|}
\hline \multirow{2}{*}{$\begin{array}{r}\text { Case Reports in } \\
\text { Gastroenterology }\end{array}$} & \multicolumn{2}{|c|}{ Case Rep Gastroenterol 2016;10:315-322 } & \multirow[b]{2}{*}{$\begin{array}{l}\text { Karger } \\
\text { Open access }\end{array}$} \\
\hline & $\begin{array}{l}\text { DOI: 10.1159/000447290 } \\
\text { Publisned onine: June 27, } 2016\end{array}$ & $\begin{array}{l}\text { (c) } 2016 \text { The Author(s) } \\
\text { Published by S. Karger AG, Basel } \\
\text { www.karger.com/crg }\end{array}$ & \\
\hline & $\begin{array}{l}\text { This article is licensed under } \\
\text { International License (CC BY-N } \\
\text { Usage and distribution for comm }\end{array}$ & $\begin{array}{l}\text { nons Attribution-NonCommercia } \\
\text { ger.com/Services/OpenAccessLice } \\
\text { uires written permission. }\end{array}$ & \\
\hline
\end{tabular}

\title{
Efficacy of Prothrombin Complex Concentrate Treatment in Patients with Liver Coagulopathy Who Underwent Various Invasive Hepatobiliary and Gastrointestinal Procedures
}

\author{
Cosmas Rinaldi A. Lesmana ${ }^{a, b} \quad$ Lidwina Cahyadinata $^{a} \quad$ Levina S. Pakasi $^{a}$ \\ Laurentius A. Lesmana ${ }^{a, b}$ \\ ${ }^{a}$ Digestive Disease \& GI Oncology Center, Medistra Hospital, Jakarta, Indonesia; \\ ${ }^{\mathrm{b}}$ Hepatobiliary Division, Department of Internal Medicine, Cipto Mangunkusumo Hospital, \\ University of Indonesia, Jakarta, Indonesia
}

\section{Keywords}

International normalized ratio · Liver cirrhosis · Liver coagulopathy · Prothrombin complex concentrate

\begin{abstract}
Background: Prothrombin complex concentrates (PCCs) containing prothrombin, factors VII, IX, and $X$, as well as the inhibitors protein $C$ and $S$ have been used as an emergent reversal for oral anticoagulation therapy. The use of PCCs in hepatobiliary disorder patients or patients with liver coagulopathy who need to undergo invasive procedures has not been well studied. Objective: To evaluate the efficacy of PCC treatment in order to control or prevent bleeding complications in patients with liver coagulopathy who undergo various invasive procedures. Methods: This was a prospective, open-label, non-randomized, before-and-after study in patients with hepatobiliary disorders who underwent invasive procedures accompanied by liver impairment and received PCC injection (Cofact ${ }^{\circledR}$, Sanquin, The Netherlands). Patients with coagulopathy from various causes were recruited consecutively. Data collected
\end{abstract}


were the episodes of bleeding, liver function test and the international normalized ratio (INR) before and after PCC therapy. The primary endpoint was INR change after treatment, while secondary endpoints included bleeding control and bleeding event after treatment. Results: Thirty patients (17 men, 13 women) were enrolled. Patients' mean age was $57.0+15.5$ years. Liver cirrhosis was found in 14 patients (46.7\%). The procedures consisted of liver biopsy, liver abscess aspiration, abdominal paracentesis, therapeutic upper gastrointestinal endoscopy, abdominal surgery, endoscopic retrograde cholangiopancreatography and percutaneous transhepatic biliary drainage. After treatment, 25 patients (83.3\%) showed a decreased median INR (from 1.6 to 1.3) ( $p<0.001$, Wilcoxon's signed-rank test). Five patients failed to show INR reduction. No new bleeding event related to the invasive procedures was observed. Conclusion: PCC treatment is effective to control and prevent bleeding complications in patients with liver coagulopathy who undergo invasive procedures.

(C) 2016 The Author(s)

Published by S. Karger AG, Basel

\section{Introduction}

Most factors involved in the blood clotting and fibrinolytic systems are synthesized in the liver, and disturbed liver parenchymal cell function may result in serious impact on the blood coagulation system. The severity of blood coagulopathy is associated with the degree of liver damage and may be severe in patients with cirrhosis [1].

Patients with chronic liver disease or chronic cholestasis often have a tendency for spontaneous bleeding, including variceal bleeding due to portal hypertension and low levels of coagulation factors. In addition, there is an increased perioperative bleeding risk, including minor invasive diagnostic procedures like liver biopsy. The total bleeding rate ranges between 0.7 and $1.5 \%$ in patients with liver disease [2,3].

Prothrombin complex concentrates (PCCs) are plasma products containing mainly the plasma-clotting factors prothrombin, factors VII, IX, and X, the inhibitors protein C and S, and protein Z [4]. They are produced from the cryoprecipitate supernatant of large plasma pools or from the plasma supernatant after the removal of antithrombin and factor XI.

PCC therapy is used as a single-factor replacement in conditions such as hemophilia B, but currently it is used mainly for emergent reversal the effects of oral anticoagulation therapy in an emergency setting [5, 6]. However, the use of PCCs to prevent bleeding in patients with liver coagulopathy has not been studied well, especially in those patients who undergo various hepatobiliary and gastrointestinal therapeutic intervention procedures. The major reasons are the cost and the controversy among hematologists related to liver coagulopathypathophysiology.

The international normalized ratio (INR) has been incorporated into several scoring systems to assess liver disease severity in cirrhosis patients, such as the Child-Turcotte-Pugh and the Model for End-Stage Liver Disease scores [7]. These tools are highly associated with the patient's prognosis, but there is no recommendation on how to relate these scoring systems in assessing bleeding risk.

The aim of this study was to evaluate the efficacy of PCC treatment in order to control bleeding or prevent bleeding in patients with liver coagulopathy scheduled for various invasive procedures. 


\section{Methods}

\section{Study Design and Subjects}

This was a prospective, open-label, non-randomized study in patients who received PCC injection (Cofact ${ }^{\circledR}$, Sanquin, The Netherlands), consisting of coagulation factors II, VII, IX, and $\mathrm{X}$. Patients were recruited consecutively. Data collected were the episodes of bleeding, liver function test and the INR calculated before and $1 \mathrm{~h}$ after injection.

\section{Diagnosis of Liver Coagulopathy}

In our institution, a diagnosis of liver coagulopathy was made if the INR value was $\geq 1.4$. INR calculation was based on the patient's prothrombin time in seconds divided by normal prothrombin time (the laboratory's geometric mean value for normal patients) in seconds, and the results were powered by the International Sensitivity Index.

\section{Study Medication and Dosage}

PCC treatment is given as a single injection $1 \mathrm{~h}$ before a particular invasive procedure. The contents of a vial are 250 IU factor IX, 140-350 IU factor II, 70-200 IU factor VII, 140350 IU factor X, 111-390 IU protein C and 10-80 IU protein S. After dilution with the presupplied water for injection, the final solution contains $14-35 \mathrm{IU} / \mathrm{ml}$ factor II, 7-20 IU/ml factor VII, $25 \mathrm{IU} / \mathrm{ml}$ factor IX, 14-35 IU/ml factor X, 11-39 IU/ml protein C, and 1-8 IU/ml protein $\mathrm{S}$.

\section{Treatment Efficacy}

As primary endpoint, INR was calculated during $24 \mathrm{~h}$ before and $1 \mathrm{~h}$ after the administration of PCCs. Secondary endpoints were bleeding control and bleeding event after treatment. Bleeding control was defined as bleeding cessation after treatment. Bleeding event was defined as active bleeding after the invasive procedure which was seen clinically or by a decrease in hemoglobin level $>1 \mathrm{~g} / \mathrm{dl} 24 \mathrm{~h}$ after treatment.

\section{Statistical Analyses}

The patients' demographic and clinical characteristics were presented descriptively. The median INR difference between before and after PCC treatment was analyzed using the Wilcoxon's signed-rank test. A p value $\leq 0.05$ was considered significant. Statistical analyses were done using the software SPSS version 17.0 (SPSS Inc., Chicago, Ill., USA).

\section{Results}

\section{Patient Characteristics}

Thirty patients were enrolled in this study, consisting of 17 men and 13 women. Patients' mean age was $57.0 \pm 15.5$ years. The liver function test profiles are summarized in table 1. Liver cirrhosis was found in 14 patients (46.7\%). The procedures consisted of liver biopsy, liver abscess aspiration, large-volume abdominal paracentesis, therapeutic upper gastrointestinal endoscopy (band ligation, Histoacryl injection, hemostatic), abdominal surgery, endoscopic retrograde cholangiopancreatography and percutaneous transhepatic biliary drainage. The underlying diseases causing liver coagulopathy are summarized in table 2. 


\section{Efficacy of PCC Treatment}

Twenty-five (83.3\%) patients showed a decreased INR after PCC injection as given according to the dose recommendation (table 3). In these patients, the median (range) INR decreased from $1.6(1.4-4.7)$ to $1.3(0.9-2.4), \mathrm{p}<0.001$ (fig. 1). Fifteen $(60.0 \%)$ of them achieved an INR $<1.4$.

There were 5 patients who failed to show INR reduction, 2 patients with severe bleeding, 1 patient with decompensated cirrhosis, and in the last 2 patients it might have been related to the delayed INR measurement, as it exceeded $6 \mathrm{~h}$ after the PCC injection.

Nine patients presented with active bleeding before treatment. Bleeding was controlled in 5 patients; 3 patients responded after receiving 1 PCC vial, 1 patient received 2 PCC vials, and the other patient received until 9 vials. Of the 4 patients with continuous bleeding, 2 patients received only 1 PCC vial, while the other 2 patients received 2 and 3 PCC vials. Despite the INR reduction (although it was still high) in those patients, 2 critically ill patients had severe stomach bleeding (due to gastric malignancy) and died because of hypovolemic shock.

\section{Discussion}

To our knowledge, this is the first study in Asia looking at the impact of PCC treatment in liver coagulopathy patients who underwent various intervention procedures in the field of gastroenterology.

This study was designed to evaluate whether PCC treatment could prevent bleeding in patients with liver coagulopathy who underwent urgent medical procedures. Diagnoses in these patients included chronic liver diseases (chronic hepatitis, liver cirrhosis, hepatocellular carcinoma), acute hepatobiliary diseases (liver abscess, gallstone, acute cholangitis) as well as non-liver diseases (gastric cancer). Moreover, these patients were not on anticoagulation treatment; therefore, the high INR was caused solely by liver derangement.

Liver coagulopathy is caused by hepatic dysfunction of coagulation factor synthesis and cannot be treated by the administration of vitamin $\mathrm{K}$.

Traditionally, hypercoagulated patients with decompensated liver cirrhosis were given fresh frozen plasma (FFP) transfusion, despite unproven efficacy or lack of benefit of its use [8]. In addition, FFP is often given in inadequate doses and therefore rarely achieves the INR target [9]. FFP transfusion is also associated with fluid overload risk as it sometimes requires several liters of FFP (20-40 ml/kg) to achieve the INR target [10].

One study reported the use of FFP versus PCCs in reversing anticoagulation therapy in patients undergoing cardiopulmonary bypass surgery with an INR $\geq 2.1$. In that study, the investigators used 2 units of FFP and half the dose of PCCs to achieve a target INR of $\leq 1.5$. They found that the INR target can be achieved in 7/16 patients with PCCs, but in none with FFP 15 min after surgery, but after $1 \mathrm{~h}$, both treatments can achieve the INR target. Overall, the study concluded that PCCs reverse the anticoagulation effect faster and with less bleeding than FFP [11].

Another recent study also confirmed the superiority of 4-factor PCCs versus plasma transfusion to achieve an INR $\leq 1.3$ in terms of rapidity. One hour after the start of infusion, $69 \%$ of patients in the PCC group achieve an INR $\leq 1.3$, compared with none in the plasma transfusion group. In addition, the median INR was significantly lower in patients treated with PCCs compared with the plasma transfusion group [12]. 
There were some limitations in this study. First, it was not designed as a randomized, controlled study to compare the treatment effect. The use of PCC injection in our study was intended to substitute FFP as these patients need to undergo urgent procedures. It is also well known that FFP transfusion is rarely effective in reducing INR within a relatively short time. Therefore, comparing PCCs with FFP or placebo as a control would not have been ethical. Besides, there was no other treatment option as a control in this clinical setting. Before PCCs were available in our country, emergency procedures were mostly delayed or even could not be done if the patient did not show INR reduction after standard treatment using vitamin $\mathrm{K}$ injection with or without plasma transfusion. Second, since this is a preliminary study, we did not specifically select more homogeneous patients and included a wide range of underlying diseases. This caused difficulty in analyzing risk factors that may contribute to treatment failure or non-responsiveness. It seems that patients with active severe bleeding may not gain much benefit from 1 or 2 vials of PCC treatment. However, the exact doses for these patients remain unclear. Our study addresses an important message to clinicians, despite the clinical dilemma of cost effectiveness, especially in most developing countries. Decompensated liver failure may also worsen the prognosis, and adding PCC treatment seemed to show no additional benefit in terms of prolonging life. These assumptions should be explored in further studies.

\section{Conclusion}

PCC treatment is effective in reducing the INR and to prevent bleeding in patients with liver coagulopathy undergoing urgent or emergency invasive procedures. Patients with severe liver function derangement or severe bleeding may benefit from INR reduction; however, survival benefits should be further defined.

\section{Statement of Ethics}

All subjects gave written consent before the administration of PCC treatment. This study was approved by the local ethics committee.

\section{Disclosure Statement}

The authors declare no conflicts of interest.

\section{References}

1 Mammen EF: Coagulopathies of liver disease. Clin Lab Med 1994;14:769-780.

- Eve K: Bleeding after liver biopsy does not correlate with indices of peripheral coagulation. Dig Dis Sci 1981;256:388-393.

3 Mahal AS, Knauer CM, Gregory PB: Bleeding after liver biopsy. West J Med 1981;134:11-14.

4 Hellstern P, Halbmayer WM, Köhler M, Seitz R, Müller-Berghaus G: Prothrombin complex concentrates: indications, contraindications, and risks: a task force summary. Thromb Res 1999;95(4 suppl 1):S3-S6.

5 Ansell J, Hirsh J, Hylek E, Jacobson A, Crowther M, Palareti G: Pharmacology and management of the vitamin K antagonists: American College of Chest Physicians Evidence-Based Clinical Practice Guidelines (8th edition). Chest 2008;133(6 suppl):160S-198S. 
6 Baglin TP, Keeling DM, Watson HG: Guidelines on oral anticoagulation (warfarin): third edition - 2005 update. Br J Haematol 2006;132:277-285.

7 Malinchoc M, Kamath PS, Gordon FD, Peine CJ, Rank J, ter Borg PC: A model to predict poor survival in patients undergoing transjugular intrahepatic portosystemic shunts. Hepatology 2000;31:864-871.

$>8$ Shah NL, Northyp PG, Caldwell SH: A clinical survey of bleeding, thrombosis, and blood product use in decompensated cirrhosis patients. Ann Hepatol 2012;11:686-690.

-9 Youssef WI, Salazar F, Dasarathy S, Beddow T, Mullen KD: Role of fresh frozen plasma infusion in correction of coagulopathy of chronic liver disease: a dual phase study. Am J Gastroenterol 2003;98: 1391-1394.

10 Holland LL, Brooks JP: Toward rational fresh frozen plasma transfusion: the effect of plasma transfusion on coagulation test results. Am J Clin Pathol 2006;126:133-139.

11 Demeyere R, Gillardin S, Arnout J, Strengers PFW: Comparison of fresh frozen plasma and prothrombin complex concentrate for the reversal of oral anticoagulants in patients undergoing cardiopulmonary bypass surgery: a randomized study. Vox Sang 2010;99:251-260.

12 Sarode R, Milling TJ Jr, Refaai MA, Mangione A, Schneider A, Durn BL, Goldstein JN: Efficacy and safety of a 4-factor prothrombin complex concentrate in patients on vitamin $\mathrm{K}$ antagonists presenting with major bleeding: a randomized, plasma-controlled, phase IIIb study. Circulation 2013;128:1234-1243.

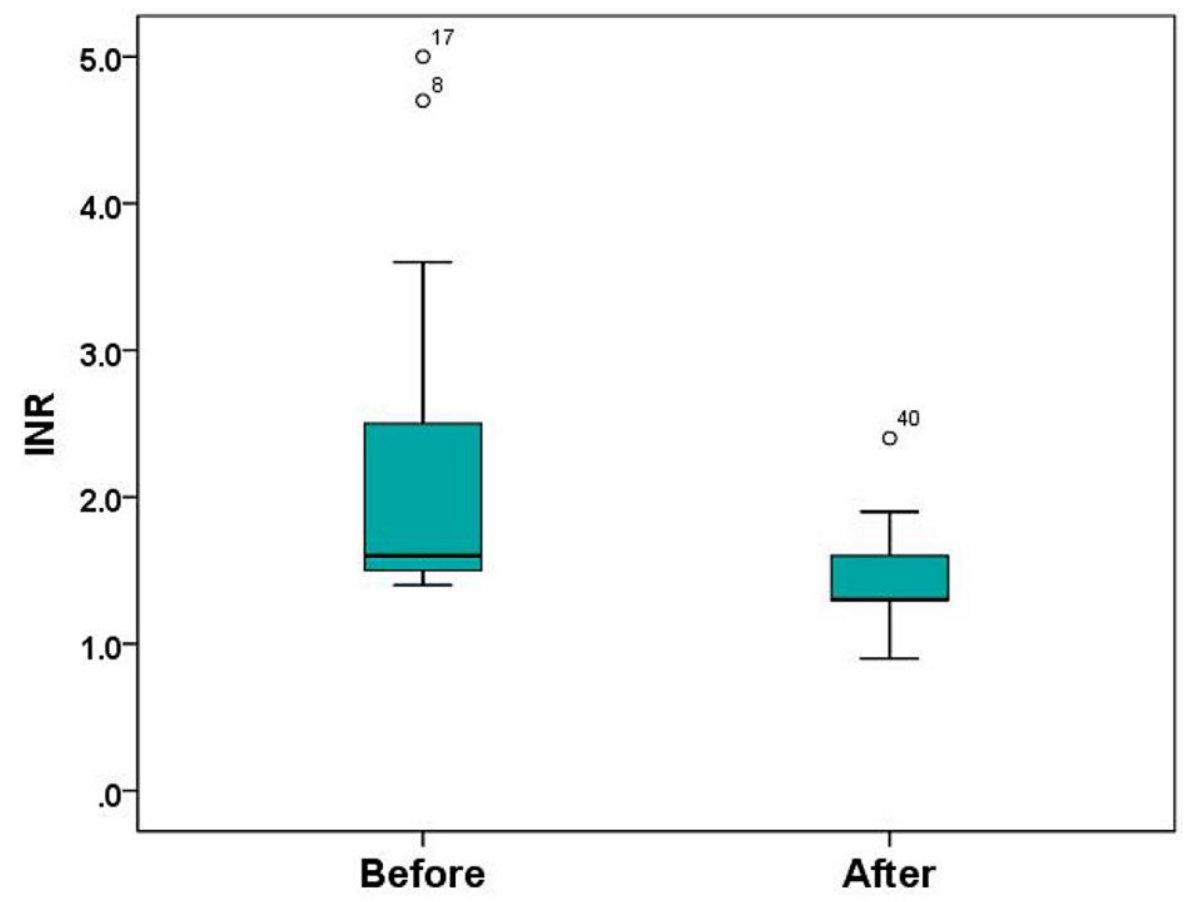

Fig. 1. The mean difference of the INR values between before and after PCC injection $(n=25)$. 
Lesmana et al:: Efficacy of PCC Treatment in Patients with Liver Coagulopathy Who Underwent Various Invasive Hepatobiliary and Gastrointestinal Procedures

Table 1. Characteristics of the study subjects $(n=30)$

\begin{tabular}{lccc}
\hline Variable & Median (range) & $\mathrm{n}$ & $\%$ \\
\hline Male sex & & 17 & 56.7 \\
Age, years & & & \\
Active bleeding & & & 30.0 \\
$\quad$ Peripheral blood test & & & \\
$\quad$ Hemoglobin level, g/dl & $10.9(6.3-15.6)$ & & \\
$\quad$ Leukocyte count, /ml & $11,865(1,300-29,500)$ & & \\
$\quad$ Platelet count, $/ \mathrm{nl}$ & $179(24-698)$ & & \\
Liver function test & & & \\
$\quad$ Total bilirubin, mg/dl & $3.38(0.3-51.95)$ & & \\
$\quad$ Total protein, g/dl & $6.2(5.1-7.6)$ & & \\
$\quad$ Albumin, g/dl & $3.0(1.8-4.5)$ & & \\
AST, U/l & $62(13-293)$ & & \\
ALT, U/l & $45.5(10-162)$ & & \\
GGT, U/l & $117(11-1,151)$ & & \\
$\quad$ ALP, U/l & $159(64-760)$ & & \\
INR & $1.6(1.4-5.0)$ & & \\
\hline
\end{tabular}

ALP = Alkaline phosphatase; ALT = alanine aminotransferase; AST = aspartate aminotransferase; GGT = gamma-glutamyl transferase. 
Table 2. Underlying disease and indication for PCC treatment $(n=30)$

\begin{tabular}{lrr}
\hline & $\mathrm{n}$ & $\%$ \\
\hline Underlying disease & & 16.7 \\
Liver abscess & 5 & 36.7 \\
Liver cirrhosis & 11 & 3.3 \\
Liver cirrhosis and abscess & 1 & 3.3 \\
Liver cirrhosis and choledocholithiasis & 1 & 3.3 \\
Liver cirrhosis and hepatocellular carcinoma & 1 & 3.3 \\
Hepatocellular carcinoma & 1 & 13.3 \\
Other cancers with jaundice/liver metastasis & 4 & 3.3 \\
Choledocholithiasis with obstructive jaundice & 1 & 3.3 \\
Pelvic inflammatory disease and appendiceal abscess & 1 & 3.3 \\
Acute-on-chronic liver failure (due to autoimmune hepatitis) & 3.3 \\
Cholangitis and acute kidney injury & 1 & 6.7 \\
Autoimmune hepatitis with jaundice & 1 & 16.7 \\
\hline Indication for PCC treatment & 2 & 3.3 \\
Abscess aspiration & & 26.7 \\
Large-volume abdominal paracentesis & 5 & 30.0 \\
Other hepatobiliary procedures (ERCP, PTBD) & 1 & 10.0 \\
Gastrointestinal bleeding & 8 & 10.0 \\
Liver biopsy & 9 & 3.3 \\
Open surgery & 3 & 3 \\
Laparoscopic surgery & 1 & \\
\hline
\end{tabular}

ERCP = Endoscopic retrograde cholangiopancreatography; PTBD = percutaneous transhepatic biliary drainage.

Table 3. The recommended dose (ml) of PCC injection to achieve a target INR of $\leq 1.5$ is based on the initial INR and body weight as follows

\begin{tabular}{|c|c|c|c|c|c|c|c|c|c|c|c|c|}
\hline \multirow[t]{2}{*}{ Body weight } & \multicolumn{12}{|c|}{ Initial INR } \\
\hline & 7.5 & 5.9 & 4.8 & 4.2 & 3.6 & 3.3 & 3.0 & 2.8 & 2.6 & 2.5 & 2.3 & 2.2 \\
\hline $50 \mathrm{~kg}$ & 60 & 60 & 60 & 50 & 50 & 50 & 40 & 40 & 30 & 30 & 30 & 30 \\
\hline $60 \mathrm{~kg}$ & 80 & 70 & 70 & 60 & 60 & 60 & 50 & 50 & 40 & 40 & 40 & 30 \\
\hline $70 \mathrm{~kg}$ & 90 & 80 & 80 & 70 & 70 & 70 & 60 & 60 & 50 & 40 & 40 & 40 \\
\hline $80 \mathrm{~kg}$ & 100 & 100 & 90 & 90 & 90 & 80 & 80 & 70 & 60 & 50 & 50 & 40 \\
\hline $90 \mathrm{~kg}$ & 100 & 100 & 100 & 90 & 90 & 90 & 80 & 80 & 70 & 60 & 50 & 40 \\
\hline $100 \mathrm{~kg}$ & 100 & 100 & 100 & 100 & 100 & 90 & 90 & 80 & 70 & 70 & 60 & 50 \\
\hline
\end{tabular}

